\title{
On the Liouville property for fully nonlinear equations *
}

by

\author{
Alessandra CUTRÌ ${ }^{\mathrm{a}}$, Fabiana LEONI ${ }^{\mathrm{b}}$ \\ a Dipartimento di Matematica, Università di Roma, "Tor Vergata" 00133 Roma, Italy \\ b Dipartimento di Matematica, Università di Roma, "La Sapienza" 00185 Roma, Italy \\ Manuscript received 1 December 1998
}

ABSTRACT. - In this paper we prove some Liouville theorems for nonnegative viscosity supersolutions of a class of fully nonlinear uniformly elliptic problems in $\mathbb{R}^{N}$.

(C) 2000 L'Association Publications de l'Institut Henri Poincaré. Published by Elsevier B.V. All rights reserved

RÉSUMÉ. - Dans ce travail nous démontrons des théorèmes de Liouville pour des sur-solutions de viscosité positives de problèmes uniformement elliptique complètement non linéaires dans $\mathbb{R}^{N}$.

(C) 2000 L'Association Publications de l'Institut Henri Poincaré. Published by Elsevier B.V. All rights reserved

\section{INTRODUCTION}

The aim of this paper is to prove the Liouville property for nonnegative viscosity supersolutions of a class of fully nonlinear uniformly elliptic equations in the whole space $\mathbb{R}^{N}$. We consider problems of the form

$$
u \geqslant 0, \quad \mathcal{M}_{\lambda, \Lambda}^{+}\left(D^{2} u\right) \leqslant 0 \quad \text { in } \mathbb{R}^{N},
$$

\footnotetext{
* This work was partially supported by the TMR Network "Viscosity Solutions and Applications".
} 
where $\mathcal{M}_{\lambda, \Lambda}^{+}$, for fixed $0<\lambda \leqslant \Lambda$, is the Pucci extremal operator

$$
\mathcal{M}_{\lambda, \Lambda}^{+}\left(D^{2} u\right)=\sup _{A \in \mathcal{A}_{\lambda, \Lambda}} \operatorname{tr}\left(A D^{2} u\right)
$$

with

$$
\mathcal{A}_{\lambda, \Lambda}=\left\{A \in \mathcal{S}_{N}: \lambda|\xi|^{2} \leqslant A \xi \cdot \xi \leqslant \Lambda|\xi|^{2}, \forall \xi \in \mathbb{R}^{N}\right\}
$$

( $\mathcal{S}_{N}$ denotes the space of all real symmetric $N \times N$ matrices), or of the form

$$
u \geqslant 0, \quad F\left(x, D^{2} u\right)+h(x) u^{p} \leqslant 0 \quad \text { in } \mathbb{R}^{N},
$$

where $F$ is a uniformly elliptic operator with ellipticity constants $0<\lambda \leqslant$ $\Lambda$. Precisely, we assume that $F: \mathbb{R}^{N} \times \mathcal{S}_{N} \rightarrow \mathbb{R}$ is a continuous function satisfying, for some $0<\lambda \leqslant \Lambda$, the following conditions:

$$
\lambda \operatorname{tr}(P) \leqslant F(x, M+P)-F(x, M) \leqslant \Lambda \operatorname{tr}(P)
$$

for all $M, P \in \mathcal{S}_{N}$ with $P \geqslant 0$ (i.e. nonnegative definite) and

$$
F(x, 0) \equiv 0 \quad \text { for all } x \in \mathbb{R}^{N} .
$$

For problem (1.1) we prove that $u$ is necessarily a constant, provided that $N \leqslant 1+\frac{\Lambda}{\lambda}$ (see Theorem 3.2).

On the other hand for problem (1.2), under some restrictions on $h$ and $p$ (see Theorem 4.1), the only solution is $u \equiv 0$.

Note that in the case $\lambda=\Lambda=1$ the operator $\mathcal{M}_{\lambda, \Lambda}^{+}$reduces to the Laplace operator so that the first result generalizes the well-known Liouville property for nonnegative superharmonic function in $\mathbb{R}^{N}$, with $N \leqslant 2$.

A major step in the proof of Theorem 3.2 is to establish a convexity result for viscosity solutions of (1.1) in the spirit of the Hadamard three circles theorem (see Theorem 3.1).

The result of Theorem 3.2 is optimal: there are examples of nontrivial solutions of (1.1) if $N>1+\frac{\Lambda}{\lambda}$ (see Remark 2).

Moreover, for a general fully nonlinear uniformly elliptic operator $F: \mathbb{R}^{N} \times \mathcal{S}_{N} \rightarrow \mathbb{R}$, the problem

$$
u \geqslant 0, \quad F\left(x, D^{2} u\right) \leqslant 0 \quad \text { in } \mathbb{R}^{N},
$$

with $N \geqslant 2$, may have nonconstant viscosity solutions (see Remark 3 ). Let us observe at this purpose that, in the case of equality, the Liouville 
property holds true: the constant functions are the only bounded either from above or from below viscosity solutions of

$$
F\left(x, D^{2} u\right)=0 \quad \text { in } \mathbb{R}^{N}
$$

This result can be found in [8]; its proof relies on the Krylov-SafonovHarnack inequality (see also [15]).

The fully nonlinear problem (1.2) will be considered in our Section 4: in Theorem 4.1 we obtain the Liouville property assuming that $h$ is a nonnegative continuous function on $\mathbb{R}^{N}$ satisfying the growth condition

$$
h(x) \geqslant H|x|^{\gamma} \quad \text { if }|x| \geqslant r_{0}
$$

for some constants $r_{0}, H>0$ and $\gamma \geqslant-2$ and provided that the exponent $p$ satisfies

$$
0<p \leqslant \frac{\frac{\Lambda}{\lambda}(N-1)+1+\gamma}{\frac{\Lambda}{\lambda}(N-1)-1} \text { if } \gamma>-2 \text {, }
$$

and

$$
0<p<1 \text { if } \gamma=-2
$$

Let us recall that the semilinear case

$$
u \geqslant 0, \quad \Delta u+h(x) u^{p} \leqslant 0 \quad \text { in } \mathbb{R}^{N},
$$

has been already treated in the case $p>1$ and $\gamma>-2$ (see $[2,3,6,9,10])$ : it is known that if

$$
1<p \leqslant \frac{N+\gamma}{N-2}
$$

then zero is the only solution, as well as if $p>(N+\gamma) /(N-2)$ then there exists a nontrivial solution (see $[6,10]$ for a counterexample). We notice that, setting $\beta=\frac{\Lambda}{\lambda}(N-1)+1$, condition (1.5) reads as

$$
0<p \leqslant \frac{\beta+\gamma}{\beta-2} \text { if } \gamma>-2
$$

it is then clear the analogy between (1.8) and (1.9) and their consistency, being $\beta=N$ in the case $\Lambda=\lambda$. Moreover we consider the cases $0<p<$ 1 and $\gamma=-2$. 
We also prove that the result is optimal: adapting the counterexample produced in [10], we show the existence of nontrivial solutions of (1.2) (for a particular $F$ ) in the cases

$$
p>\frac{\frac{\Lambda}{\lambda}(N-1)+1+\gamma}{\frac{\Lambda}{\lambda}(N-1)-1} \quad \text { when } \gamma>-2,
$$

$p \geqslant 1$ when $\gamma=-2$ and $p>0$ when $\gamma<-2$.

Let us finally remark that the Liouville property for semilinear elliptic and degenerate elliptic equations, posed in the whole space $\mathbb{R}^{N}$ or in cones or halfspaces, has been the object of a keen interest in the literature also for its connection with the problem of the a priori bounds and the existence of positive solutions of superlinear boundary value problems in bounded domains. The first results in this direction are contained in $[13,14]$ in which the semilinear uniformly elliptic equation in $\mathbb{R}^{N}$ and in halfspaces is considered; under different assumptions, analogous results for the equation have been subsequently obtained also in [1,22]. Again the equation but in an elliptic degenerate case is considered in [18,24,25]. The inequality in the whole space and in cones has been treated in $[2,3,6]$ and in $[4,5,10,12]$ for some elliptic degenerate cases. Anyway, we refer to [9] for a general overview on this subject. The extension of these results to the fully nonlinear case will be the subject of a forthcoming paper.

\section{PRELIMINARIES}

In this section we recall some basic notions and known results about fully nonlinear elliptic equations. For further details we refer to, e.g., [8, $11]$.

Here and in the sequel $\mathcal{S}_{N}$ denotes the set of all symmetric $N \times N$ matrices, and the dimension $N$ will be always assumed to satisfy $N \geqslant 2$.

A continuous function $F: \mathbb{R}^{N} \times \mathcal{S}_{N} \rightarrow \mathbb{R}$ will be referred to as a uniformly elliptic operator with ellipticity constants $0<\lambda \leqslant \Lambda$ if, for all $M, P \in \mathcal{S}_{N}$ with $P \geqslant 0$ (i.e., nonnegative definite), and for all $x \in \mathbb{R}^{N}$, it results

$$
\lambda \operatorname{tr}(P) \leqslant F(x, M+P)-F(x, M) \leqslant \Lambda \operatorname{tr}(P) .
$$

In the rest of the paper we will always consider uniformly elliptic operators $F(x, M)$ such that

$$
F(x, 0) \equiv 0
$$


The simplest example of operator $F$ satisfying (2.1) (and (2.2)) is the linear map

$$
\mathcal{L}_{A}(x, M) \equiv \mathcal{L}_{A}(M)=\operatorname{tr}(A M),
$$

where $A \in \mathcal{S}_{N}$ is a positive definite matrix the eigenvalues of which belong to $[\lambda, \Lambda]$.

Let us indicate with $\mathcal{A}_{\lambda, \Lambda}$ the set of all such matrices $A$, i.e.

$$
\mathcal{A}_{\lambda, \Lambda}=\left\{A \in \mathcal{S}_{N}: \lambda|\xi|^{2} \leqslant A \xi \cdot \xi \leqslant \Lambda|\xi|^{2}, \forall \xi \in \mathbb{R}^{N}\right\}
$$

Since the family of uniformly elliptic operators having common ellipticity constants is closed under the sup or the inf process, the definitions

$$
\mathcal{M}_{\lambda, \Lambda}^{-}(M)=\inf _{A \in \mathcal{A}_{\lambda, \Lambda}} \mathcal{L}_{A}(M), \quad \mathcal{M}_{\lambda, \Lambda}^{+}(M)=\sup _{A \in \mathcal{A}_{\lambda, \Lambda}} \mathcal{L}_{A}(M),
$$

produce other two significant examples of uniformly elliptic operators, called extremal operators (see $[7,8]$ ), related by the identity

$$
\mathcal{M}_{\lambda, \Lambda}^{+}(M)=-\mathcal{M}_{\lambda, \Lambda}^{-}(-M) \quad \forall M \in \mathcal{S}_{N}
$$

Slightly different extremal operators have been firstly introduced by Pucci in [20], where the inf and sup are taken on the class $\mathcal{B}_{\alpha}, \alpha>0$, defined as

$$
\mathcal{B}_{\alpha}=\left\{A \in \mathcal{S}_{N}: A \xi \cdot \xi \geqslant \alpha|\xi|^{2}, \operatorname{tr} A=1, \forall \xi \in \mathbb{R}^{N}\right\}
$$

(see also [15]). Thus, the Pucci extremal operators are defined as

$$
\mathcal{P}_{\alpha}^{-}(M)=\inf _{A \in \mathcal{B}_{\alpha}} \mathcal{L}_{A}(M), \quad \mathcal{P}_{\alpha}^{+}(M)=\sup _{A \in \mathcal{B}_{\alpha}} \mathcal{L}_{A}(M)
$$

Observing that if $A$ belongs to $\mathcal{A}_{\lambda, \Lambda}$ then the normalized matrix $\frac{1}{\operatorname{tr} A} A$ belongs to $\mathcal{B}_{\lambda /(\Lambda N)}$ and, conversely, if $A$ belongs to $\mathcal{B}_{\alpha}$ then $A$ belongs to $\mathcal{A}_{\alpha, 1-(N-1) \alpha}$, it results

$$
\mathcal{M}_{\lambda, \Lambda}^{+}(M) \leqslant N \Lambda \mathcal{P}_{\lambda /(\Lambda N)}^{+}(M), \quad \mathcal{P}_{\alpha}^{+}(M) \leqslant \mathcal{M}_{\alpha, 1-(N-1) \alpha}^{+}(M)
$$

and, analogously,

$$
\mathcal{M}_{\lambda, \Lambda}^{-}(M) \geqslant N \lambda \mathcal{P}_{\lambda /(\Lambda N)}^{-}(M), \quad \mathcal{P}_{\alpha}^{-}(M) \geqslant \mathcal{M}_{\alpha, 1-(N-1) \alpha}^{-}(M)
$$


In the sequel we will always deal with the extremal operators $\mathcal{M}_{\lambda, \Lambda}^{+}$and $\mathcal{M}_{\lambda, \Lambda}^{-}$, even if the previous inequalities show that every argument could be carried out for the Pucci operators $\mathcal{P}_{\alpha}^{+}$and $\mathcal{P}_{\alpha}^{-}$, with $\alpha$ appropriately chosen.

It is not hard to check that the operators $\mathcal{M}_{\lambda, \Lambda}^{-}$and $\mathcal{M}_{\lambda, \Lambda}^{+}$may be equivalently defined respectively as

$$
\mathcal{M}_{\lambda, \Lambda}^{-}(M)=\lambda \sum_{e_{i}>0} e_{i}+\Lambda \sum_{e_{i}<0} e_{i}
$$

and

$$
\mathcal{M}_{\lambda, \Lambda}^{+}(M)=\Lambda \sum_{e_{i}>0} e_{i}+\lambda \sum_{e_{i}<0} e_{i},
$$

where $e_{i}(i=1, \ldots, N)$ denote the eigenvalues of the matrix $M$.

The adjective "extremal" is also due to the fact that for every operator $F$ satisfying (2.1) and (2.2) with ellipticity constants $\lambda$ and $\Lambda$, it results:

$$
\mathcal{M}_{\lambda, \Lambda}^{-}(M) \leqslant F(x, M) \leqslant \mathcal{M}_{\lambda, \Lambda}^{+}(M)
$$

for all $x \in \mathbb{R}^{N}$ and $M \in \mathcal{S}_{N}$.

Next, let us recall the notion of viscosity sub and supersolutions of the equation

$$
F\left(x, u, D^{2} u\right)=0 \quad \text { in } \Omega
$$

where $F: \Omega \times \mathbb{R} \times \mathcal{S}_{N} \rightarrow \mathbb{R}$ is a continuous map with $F(x, t, M)$ satisfying (2.1) for every fixed $t \in \mathbb{R}$ and for all $x \in \Omega$, and $\Omega \subseteq \mathbb{R}^{N}$ is an open domain (for more details see, e.g., [11]).

Definition 2.1. - A continuous function $u: \Omega \rightarrow \mathbb{R}$ is a viscosity supersolution (subsolution) of (2.8) if, for all $\zeta \in C^{2}(\Omega)$ and $x_{0} \in \Omega$ such that $u-\zeta$ has a local minimum (maximum) at $x_{0}$, it results

$$
F\left(x_{0}, u\left(x_{0}\right), D^{2} \zeta\left(x_{0}\right)\right) \leqslant(\geqslant) 0 .
$$

If $u$ is a viscosity supersolution (subsolution) we also say that $u$ verifies

$$
F\left(x, u, D^{2} u\right) \leqslant(\geqslant) 0
$$

in the viscosity sense.

Finally $u$ is a viscosity solution of (2.8) if it simultaneously is a viscosity sub and supersolution. 
Let us observe that inequalities (2.7) still hold in the viscosity sense, that is if a function $u$ is a viscosity solution of $F\left(x, D^{2} u\right) \leqslant(\geqslant) 0$, with $F$ as in (2.7), then $\mathcal{M}_{\lambda, \Lambda}^{-}\left(D^{2} u\right) \leqslant 0$ (respectively, $\mathcal{M}_{\lambda, \Lambda}^{+}\left(D^{2} u\right) \geqslant 0$ ) in the viscosity sense.

In the following sections we will make use of the so called Comparison Principle and Strong Maximum Principle for viscosity solutions of the extremal operators $\mathcal{M}_{\lambda, \Lambda}^{-}$and $\mathcal{M}_{\lambda, \Lambda}^{+}$, the proofs of which can be respectively found in [16] and [8].

THEOREM 2.1 (Comparison Principle). - Let $\Omega \subset \mathbb{R}^{N}$ be a bounded open set and let $f \in C(\Omega)$; if $u_{1}$ and $u_{2}$ are respectively a super-and $a$ subsolution either of $\mathcal{M}_{\lambda, \Lambda}^{-}\left(D^{2} u\right)=f(x)$ or of $\mathcal{M}_{\lambda, \Lambda}^{+}\left(D^{2} u\right)=f(x)$ in $\Omega$ and if $u_{1} \geqslant u_{2}$ on $\partial \Omega$, then $u_{1} \geqslant u_{2}$ in $\bar{\Omega}$.

THEOREM 2.2 (Strong Maximum Principle). - Let $\Omega \subset \mathbb{R}^{N}$ be a bounded open set and let $u$ be a viscosity supersolution (subsolution) either of $\mathcal{M}_{\lambda, \Lambda}^{-}\left(D^{2} u\right)=0$ or of $\mathcal{M}_{\lambda, \Lambda}^{+}\left(D^{2} u\right)=0$ in $\Omega$. If $u$ attains its minimum (maximum) at an interior point of $\Omega$, then $u$ is constant.

\section{HADAMARD TYPE THEOREMS AND THE LIOUVILLE PROPERTY FOR EXTREMAL OPERATORS}

In this section we extend to viscosity sub- and supersolutions of the nonlinear operators $\mathcal{M}^{+}$and $\mathcal{M}^{-}$the classical Hadamard's three circles and three spheres theorems about sub- and superharmonic functions.

We recall (for more details see $[17,21]$ ) that if $u$ is a continuous superharmonic function in a plane domain containing the closed ring $\left\{x \in \mathbb{R}^{2}: r_{2} \leqslant|x| \leqslant r_{1}\right\}$, with $r_{1}>r_{2}>0$, then the Hadamard's three circles theorem states that the function $\hat{m}(r)=\min _{|x|=r} u(x)$ is a concave function of $\log r$, that is, for $r_{2} \leqslant r \leqslant r_{1}$, it satisfies

$$
\hat{m}(r) \geqslant \frac{\hat{m}\left(r_{2}\right) \log \left(r_{1} / r\right)+\hat{m}\left(r_{1}\right) \log \left(r / r_{2}\right)}{\log \left(r_{1} / r_{2}\right)} .
$$

If $u$ is a superharmonic function in a domain of $\mathbb{R}^{N}$, with $N \geqslant 3$, containing the closed ring $\left\{x \in \mathbb{R}^{N}: r_{2} \leqslant|x| \leqslant r_{1}\right\}$, then, by the Hadamard's three spheres theorem, $\hat{m}(r)$ is a concave function of $r^{2-N}$, that is, for $r_{2} \leqslant r \leqslant r_{1}$, it satisfies

$$
\hat{m}(r) \geqslant \frac{\hat{m}\left(r_{2}\right)\left(r^{2-N}-r_{1}^{2-N}\right)+\hat{m}\left(r_{1}\right)\left(r_{2}^{2-N}-r^{2-N}\right)}{r_{2}^{2-N}-r_{1}^{2-N}} .
$$


Of course, there are the "reversed" results for subharmonic functions: if $u$ is subharmonic in a plane domain, then the function $\hat{M}(r)=$ $\max _{|x|=r} u(x)$ is a convex function of $\log r$, that is, for $r_{2} \leqslant r \leqslant r_{1}$, it satisfies

$$
\hat{M}(r) \leqslant \frac{\hat{M}\left(r_{2}\right) \lg \left(r_{1} / r\right)+\hat{M}\left(r_{1}\right) \lg \left(r / r_{2}\right)}{\lg \left(r_{1} / r_{2}\right)}
$$

as well as if $u$ is subharmonic in a domain of $\mathbb{R}^{N}$, with $N \geqslant 3$, then $\hat{M}(r)$ is a convex function of $r^{2-N}$, that is, for $r_{2} \leqslant r \leqslant r_{1}$ it satisfies

$$
\hat{M}(r) \leqslant \frac{\hat{M}\left(r_{2}\right)\left(r^{2-N}-r_{1}^{2-N}\right)+\hat{M}\left(r_{1}\right)\left(r_{2}^{2-N}-r^{2-N}\right)}{r_{2}^{2-N}-r_{1}^{2-N}} .
$$

From inequality (3.1) (respectively, (3.3)), the classical Liouville's theorem easily follows, stating the nonexistence of nonconstant bounded from below (above) superharmonic (subharmonic) functions in $\mathbb{R}^{2} \backslash\{0\}$. On the contrary, it is well known that inequality (3.2) does not lead to a Liouville type theorem; indeed, for example the radial function

$$
u(x)= \begin{cases}\frac{1}{8}\left(N(N+2)-2\left(N^{2}-4\right)|x|^{2}+N(N-2)|x|^{4}\right), & \text { if }|x| \leqslant 1, \\ 1 /|x|^{N-2}, & \text { if }|x| \geqslant 1,\end{cases}
$$

is a nonconstant bounded superharmonic function in all of $\mathbb{R}^{N}, N \geqslant 3$. These different results are evidently due to the different behaviour of the fundamental solution of the Laplace equation in $\mathbb{R}^{N}$, which, as it is well known, is unbounded as $|x| \rightarrow+\infty$ if and only if $N=2$.

In order to generalize these results to the nonlinear case, first of all we have to determine the corresponding "fundamental solutions". We need the following simple technical Lemma.

Lemma 3.1. - Let $\varphi:(0,+\infty) \rightarrow \mathbb{R}$ be a $C^{2}$ function. For every $x \in \mathbb{R}^{N} \backslash\{0\}$ the eigenvalues of the Hessian matrix of the radial function $\Phi(x)=\varphi(|x|)$ are $\varphi^{\prime \prime}(|x|)$, which is simple, and $\varphi^{\prime}(|x|) /|x|$, which has multiplicity $(N-1)$.

Proof. - A direct computation shows that:

$$
D^{2} \Phi(x)=\frac{\varphi^{\prime}(|x|)}{|x|} I_{N}+\left[\frac{\varphi^{\prime \prime}(|x|)}{|x|^{2}}-\frac{\varphi^{\prime}(|x|)}{|x|^{3}}\right] x \otimes x,
$$


where $I_{N}$ is the identity matrix and $x \otimes x$ is the matrix whose entries are $x_{i} x_{j}$. Hence we have

$$
D^{2} \Phi(x) \frac{x}{|x|}=\varphi^{\prime \prime}(|x|) \frac{x}{|x|} \quad \text { and } \quad D^{2} \Phi(x) \xi=\frac{\varphi^{\prime}(|x|)}{|x|} \xi
$$

for every vector $\xi$ such that $\xi \cdot x=0$.

Using this lemma one can find, by a similar argument as in [20], radial functions $\Phi(x)=\varphi(|x|)$ which are classical solutions of the equation

$$
\mathcal{M}_{\lambda, \Lambda}^{-}\left(D^{2} \Phi\right)=0 \quad \text { in } \mathbb{R}^{N} \backslash\{0\}
$$

and are either concave and increasing or convex and decreasing.

By Lemma 3.1 and the identity (2.5), the concave and increasing functions $\varphi$ have to be looked for among the solutions of the ordinary differential equation

$$
\lambda(N-1) \frac{\varphi^{\prime}(r)}{r}+\Lambda \varphi^{\prime \prime}(r)=0 \quad \text { in }(0,+\infty)
$$

as well as the convex and decreasing solutions $\varphi$ must satisfy

$$
\lambda \varphi^{\prime \prime}(r)+\Lambda(N-1) \frac{\varphi^{\prime}(r)}{r}=0 \quad \text { in }(0,+\infty) .
$$

In both cases the solutions $\varphi$ depend on the values of the dimension $N$ and of the ellipticity constants $\lambda$ and $\Lambda$. More precisely, in the first case, setting

$$
\alpha=\frac{\lambda}{\Lambda}(N-1)+1
$$

and observing that $\alpha \geqslant 1$, we obtain the solutions

$$
\varphi_{1}(r)= \begin{cases}C_{1} r^{2-\alpha}+C_{2}, & \text { if } \alpha<2, \\ C_{1} \log r+C_{2}, & \text { if } \alpha=2, \\ -C_{1} r^{2-\alpha}+C_{2}, & \text { if } \alpha>2,\end{cases}
$$

with constants $C_{1} \geqslant 0$ and $C_{2} \in \mathbb{R}$, whereas in the second case, setting

$$
\beta=\frac{\Lambda}{\lambda}(N-1)+1
$$


and observing that $\beta \geqslant 2$ (since $N \geqslant 2$ ), the solutions are given by

$$
\varphi_{2}(r)= \begin{cases}-C_{1} \log r+C_{2}, & \text { if } \beta=2, \\ C_{1} r^{2-\beta}+C_{2}, & \text { if } \beta>2 .\end{cases}
$$

Therefore, the radial functions

$$
\Phi_{1}(x)=\varphi_{1}(|x|) \quad \text { and } \quad \Phi_{2}(x)=\varphi_{2}(|x|),
$$

with $\varphi_{1}$ and $\varphi_{2}$ respectively given by (3.7) and (3.9), are classical solutions (in particular, viscosity solutions) of Eq. (3.5).

In the following theorems they will play the same role played in the Hadamard and Liouville Theorems by the fundamental solution of the Laplace equation, and it is in this respect that they will be considered as the "fundamental solutions" of Eq. (3.5). Let us point out, moreover, that in the particular case in which $\lambda=\Lambda$, Eq. (3.5) reduces to Laplace equation; in this case, it results $\alpha=\beta=N$ and the function $\Phi_{1} \equiv \Phi_{2}$ coincides with the classical fundamental solution.

Remembering the relationship (2.3) between $\mathcal{M}_{\lambda, \Lambda}^{+}$and $\mathcal{M}_{\lambda, \Lambda}^{-}$, we have also found that the functions

$$
\Psi_{1}(x)=-\Phi_{2}(x) \quad \text { and } \quad \Psi_{2}(x)=-\Phi_{1}(x)
$$

are the "fundamental solutions" of the equation

$$
\mathcal{M}_{\lambda, \Lambda}^{+}\left(D^{2} \Psi\right)=0 \quad \text { in } \mathbb{R}^{N} \backslash\{0\},
$$

with $\Psi_{1}(x) \equiv \psi_{1}(|x|)=-\varphi_{2}(|x|)$ such that $\psi_{1}(r)$ is a concave and increasing function in $(0, \infty)$, and $\Psi_{2}(x) \equiv \psi_{2}(|x|)=-\varphi_{1}(|x|)$ such that $\psi_{2}(r)$ is a convex and decreasing function in $(0, \infty)$.

THEOREM 3.1 (Nonlinear Hadamard Theorems). - Let $D$ be a domain of $\mathbb{R}^{N}$ containing the closed ball $\bar{B}_{r_{1}}$ centered at the origin and with radius $r_{1}>0$. Then:

(i) if $u \in C(D)$ is a viscosity solution of

$$
\mathcal{M}_{\lambda, \Lambda}^{+}\left(D^{2} u\right) \leqslant 0
$$

then the function

$$
m(r)=\min _{|x| \leqslant r} u(x)
$$


is, respectively, a concave function of $\log r$ if $\alpha=2$ and of $r^{2-\alpha}$ if $\alpha \neq 2$, with $\alpha$ given by (3.6). More precisely, for every fixed $r_{2}<r_{1}$ and for all $r_{2} \leqslant r \leqslant r_{1}$, it results

$$
m(r) \geqslant \begin{cases}\frac{m\left(r_{2}\right) \log \left(r_{1} / r\right)+m\left(r_{1}\right) \log \left(r / r_{2}\right)}{\log \left(r_{1} / r_{2}\right)}, & \text { if } \alpha=2, \\ \frac{m\left(r_{2}\right)\left(r^{2-\alpha}-r_{1}^{2-\alpha}\right)+m\left(r_{1}\right)\left(r_{2}^{2-\alpha}-r^{2-\alpha}\right)}{\left(r_{2}^{2-\alpha}-r_{1}^{2-\alpha}\right)}, & \text { if } \alpha \neq 2 .\end{cases}
$$

(ii) if $u \in C(D)$ is a viscosity solution of

$$
\mathcal{M}_{\lambda, \Lambda}^{-}\left(D^{2} u\right) \leqslant 0
$$

then $m(r)$ is a concave function of $\log r$ if $\beta=2$, and of $r^{2-\beta}$ if $\beta>2$, with $\beta$ given by (3.8). More precisely, for every fixed $r_{2}<r_{1}$ and for all $r_{2} \leqslant r \leqslant r_{1}$, it results

$$
m(r) \geqslant \begin{cases}\frac{m\left(r_{2}\right) \log \left(r_{1} / r\right)+m\left(r_{1}\right) \log \left(r / r_{2}\right)}{\log \left(r_{1} / r_{2}\right)}, & \text { if } \beta=2, \\ \frac{m\left(r_{2}\right)\left(r^{2-\beta}-r_{1}^{2-\beta}\right)+m\left(r_{1}\right)\left(r_{2}^{2-\beta}-r^{2-\beta}\right)}{\left(r_{2}^{2-\beta}-r_{1}^{2-\beta}\right)}, & \text { if } \beta>2 .\end{cases}
$$

Before giving the proof of the theorem, let us observe that, by the relationship (2.3) between $\mathcal{M}_{\lambda, \Lambda}^{+}$and $\mathcal{M}_{\lambda, \Lambda}^{-}$, statement (i) is equivalent to the following one:

(j) if $u \in C(D)$ is a viscosity solution of

$$
\mathcal{M}_{\lambda, \Lambda}^{-}\left(D^{2} u\right) \geqslant 0
$$

then the function

$$
M(r)=\max _{|x| \leqslant r} u(x)
$$

satisfies, for all $r_{2} \leqslant r \leqslant r_{1}$,

$$
M(r) \leqslant \begin{cases}\frac{M\left(r_{2}\right) \log \left(r_{1} / r\right)+M\left(r_{1}\right) \log \left(r / r_{2}\right)}{\log \left(r_{1} / r_{2}\right)}, & \text { if } \alpha=2, \\ \frac{M\left(r_{2}\right)\left(r^{2-\alpha}-r_{1}^{2-\alpha}\right)+M\left(r_{1}\right)\left(r_{2}^{2-\alpha}-r^{2-\alpha}\right)}{\left(r_{2}^{2-\alpha}-r_{1}^{2-\alpha}\right)}, & \text { if } \alpha \neq 2 .\end{cases}
$$


Analogously, an equivalent form of (ii) is

(jj) if $u \in C(D)$ is a viscosity solution of

$$
\mathcal{M}_{\lambda, \Lambda}^{+}\left(D^{2} u\right) \geqslant 0
$$

then $M(r)$ satisfies, for all $r_{2} \leqslant r \leqslant r_{1}$,

$$
M(r) \leqslant \begin{cases}\frac{M\left(r_{2}\right) \log \left(r_{1} / r\right)+M\left(r_{1}\right) \log \left(r / r_{2}\right)}{\log \left(r_{1} / r_{2}\right)}, & \text { if } \beta=2, \\ \frac{M\left(r_{2}\right)\left(r^{2-\beta}-r_{1}^{2-\beta}\right)+M\left(r_{1}\right)\left(r_{2}^{2-\beta}-r^{2-\beta}\right)}{\left(r_{2}^{2-\beta}-r_{1}^{2-\beta}\right)}, & \text { if } \beta>2 .\end{cases}
$$

Proof. - By the assumptions, the respectively increasing and decreasing functions $M(r)$ and $m(r)$ are well defined in $\left[0, r_{1}\right]$.

Let us consider the case (i), that is, let $u \in C(D)$ be a viscosity solution of $\mathcal{M}_{\lambda, \Lambda}^{+}\left(D^{2} u\right) \leqslant 0$.

Fixed $r_{1}>r_{2}>0$, let $\psi(r)=-\varphi_{1}(r)$, with $\varphi_{1}(r)$ defined by (3.7), with constants $C_{1} \geqslant 0$ and $C_{2} \in \mathbb{R}$ chosen in such a way that $\psi\left(r_{1}\right)=m\left(r_{1}\right)$ and $\psi\left(r_{2}\right)=m\left(r_{2}\right)$. This yields:

$$
\psi(r)= \begin{cases}\frac{m\left(r_{2}\right) \log \left(r_{1} / r\right)+m\left(r_{1}\right) \log \left(r / r_{2}\right)}{\log \left(r_{1} / r_{2}\right)}, & \text { if } \alpha=2, \\ \frac{m\left(r_{2}\right)\left(r^{2-\alpha}-r_{1}^{2-\alpha}\right)+m\left(r_{1}\right)\left(r_{2}^{2-\alpha}-r^{2-\alpha}\right)}{\left(r_{2}^{2-\alpha}-r_{1}^{2-\alpha}\right)}, & \text { if } \alpha \neq 2 .\end{cases}
$$

We know that the function $\Psi(x)=\psi(|x|)$ is a viscosity solution of equation (3.10). Applying the Comparison Principle (Theorem 2.1) to the functions $u(x)$ and $\Psi(x)$ in the ring $\left\{r_{2}<|x|<r_{1}\right\} \subset D$, we deduce that

$$
u(x) \geqslant \Psi(x) \text { in }\left\{r_{2} \leqslant|x| \leqslant r_{1}\right\} .
$$

Hence, $m(r) \geqslant \psi(r)$ for all $r$ in $\left[r_{2}, r_{1}\right]$ and the claim is proved.

The proof of (ii) is completely analogous to that of (i), with the obvious difference that now $u$ has to be compared with the function $\Phi_{2}(x)=\varphi_{2}(|x|)$, where $\varphi_{2}$ is given by (3.9).

Looking at the previous result, as well as at the just constructed "fundamental solutions" of Eqs. (3.5) and (3.10), and having in mind the linear case, we expect a Liouville type theorem in two cases: 
- the case of bounded from below (above) viscosity supersolutions (subsolutions) of (3.10) ((3.5)) in all of $\mathbb{R}^{N}$, with the parameter $\alpha$, defined by (3.6), satisfying $\alpha \leqslant 2$,

- the case of bounded from below (above) viscosity supersolutions (subsolutions) of (3.5) ((3.10)) in all of $\mathbb{R}^{N}$, with the parameter $\beta$, defined by (3.8), satisfying $\beta=2$.

Let us observe that the case $\beta=2$ only occurs when $\lambda=\Lambda$ and $N=2$, i.e., the case of the Laplace operator in the plane.

Since viscosity subsolutions (supersolutions) of the Laplace equation are precisely the same as continuous subharmonic (superharmonic) functions (as it can be deduced, for example, from Proposition 3.2.10' of [17]), this case reduces to the well-known Liouville theorem for subharmonic (superharmonic) functions in $\mathbb{R}^{2}$.

Therefore, the first case is the only one which has to be considered.

THEOREM 3.2. - Let $u \in C\left(\mathbb{R}^{N}\right)$ be a viscosity solution either of

$$
\mathcal{M}_{\lambda, \Lambda}^{+}\left(D^{2} u\right) \leqslant 0 \quad \text { in } \mathbb{R}^{N}
$$

or of

$$
\mathcal{M}_{\lambda, \Lambda}^{-}\left(D^{2} u\right) \geqslant 0 \quad \text { in } \mathbb{R}^{N} .
$$

If $u$ is, respectively, bounded either from below or from above, and if the parameter $\alpha$, defined by (3.6), satisfies $\alpha \leqslant 2$ (i.e., $N \leqslant \frac{\Lambda}{\lambda}+1$ ), then $u$ is constant.

Proof. - Consider the case $\mathcal{M}_{\lambda, \Lambda}^{+}\left(D^{2} u\right) \leqslant 0, u$ bounded from below. By the previous theorem (case (i)), $u$ satisfies (3.11) for all $r$ in $\left[r_{2}, r_{1}\right]$, for every fixed $r_{1}>r_{2}>0$.

Being $m(r)$ a bounded function since $u$ is bounded from below, and being $\alpha \leqslant 2$, passing to the limit as $r_{1} \rightarrow+\infty$ in (3.11) leads to

$$
m(r) \geqslant m\left(r_{2}\right) \quad \forall r \geqslant r_{2}>0 .
$$

Since $m(r)$ is obviously a decreasing function, we deduce that $m(r) \equiv$ const $=m(0)=u(0)$. Therefore, $u$ attains its minimum at an interior point and, by the Strong Maximum Principle (Theorem 2.2), $u$ is constant. 
Remark 1. - The assumption on the boundedness of $u$ in the previous Theorem can be weakened: indeed, in the proof we only needed that

$$
0= \begin{cases}\limsup _{r \rightarrow+\infty} \frac{m(r)}{r^{2-\alpha}}, & \text { if } \alpha<2, \\ \limsup _{r \rightarrow+\infty} \frac{m(r)}{\log r}, & \text { if } \alpha=2 .\end{cases}
$$

Thus the Liouville Theorem for inequality (3.15) can be reformulated by saying that, for every nonconstant viscosity solution of (3.15), the function $m(r)$ decreases as $r \rightarrow+\infty$ at least like $-r^{2-\alpha}$ if $\alpha<2$ and like $-\log r$ if $\alpha=2$.

The result is optimal; indeed, the function

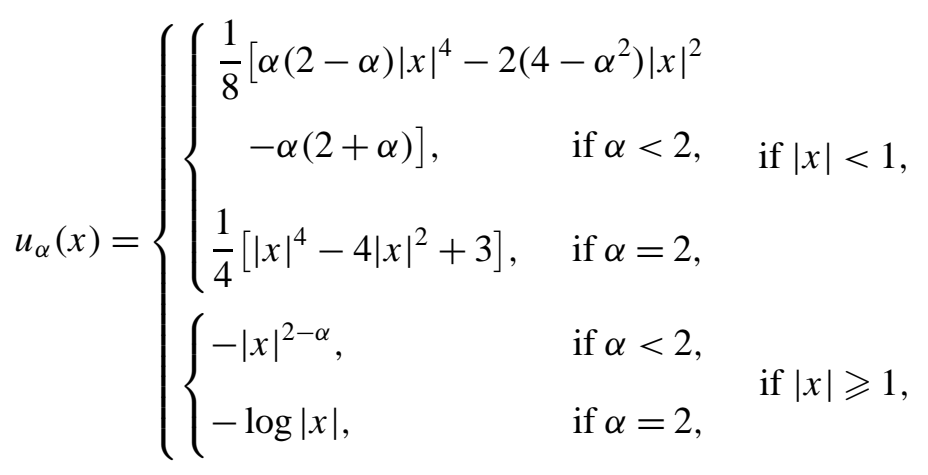

satisfies (3.15).

Remark 2. - The previous result does not hold if $\alpha>2$, i.e., if $N>$ $\frac{\Lambda}{\lambda}+1$. Indeed, in this case, the function

$$
u(x)= \begin{cases}\frac{1}{8}\left[\alpha(\alpha-2)|x|^{4}-2\left(\alpha^{2}-4\right)|x|^{2}+\alpha(\alpha+2)\right], & \text { if }|x|<1, \\ 1 /|x|^{\alpha-2}, & \text { if }|x| \geqslant 1,\end{cases}
$$

provides an example of a non constant bounded classical solution of (3.15), $-u$ obviously being a non constant bounded classical solution of (3.16).

Remark 3.-Referring to the discussion before Theorem 3.2, we observe that a Liouville type theorem does not hold in fact neither for viscosity solutions of

$$
\mathcal{M}_{\lambda, \Lambda}^{-}\left(D^{2} u\right) \leqslant 0 \quad \text { in } \mathbb{R}^{N}
$$


nor, equivalently, for viscosity solutions of

$$
\mathcal{M}_{\lambda, \Lambda}^{+}\left(D^{2} u\right) \geqslant 0 \text { in } \mathbb{R}^{N}
$$

except that for $\beta=2, \beta$ being defined in (3.8). Indeed, if $\beta>2$, then the function

$$
u(x)= \begin{cases}\frac{1}{8}\left[\beta(\beta-2)|x|^{4}-2\left(\beta^{2}-4\right)|x|^{2}+\beta(\beta+2)\right], & \text { if }|x|<1 \\ 1 /|x|^{\beta-2}, & \text { if }|x| \geqslant 1\end{cases}
$$

works as a counterexample for (3.17), as well as $-u$ does for (3.18).

Remark 4. - If (3.15) or (3.16) are required to hold as equalities, then the Liouville theorem can be obtained without any assumption on the parameter $\alpha$. More in general, for bounded either from above or from below viscosity solutions of a uniformly elliptic equation such as

$$
F\left(x, D^{2} u\right)=0 \quad \text { in } \mathbb{R}^{N}
$$

with $F$ satisfying (2.1) and (2.2), the Liouville property may be derived in a standard way as a consequence of the strong Harnack inequality, see [8].

We have seen that in the cases $\alpha, \beta>2$ a Liouville type result does not hold respectively for nonnegative viscosity solutions $u$ of (3.15) and (3.17). Nevertheless we can still deduce in such cases some important properties of the function $m(r)=\inf _{|x| \leqslant r} u(x)$. Since we are going to use them in the next section, we state the result separately.

COROLlaRY 3.1. - Let $u \in C\left(\mathbb{R}^{N}\right)$ be a viscosity solution of

$$
u \geqslant 0, \quad \mathcal{M}_{\lambda, \Lambda}^{-}\left(D^{2} u\right) \leqslant 0 \quad \text { in } \mathbb{R}^{N}
$$

(respectively of

$$
\left.u \geqslant 0, \quad \mathcal{M}_{\lambda, \Lambda}^{+}\left(D^{2} u\right) \leqslant 0 \quad \text { in } \mathbb{R}^{N}\right)
$$

then, set $\beta=\frac{\Lambda}{\lambda}(N-1)+1\left(\alpha=\frac{\lambda}{\Lambda}(N-1)+1\right)$, the function

$$
r \in[0,+\infty) \mapsto m(r) r^{\beta-2} \quad\left(r \in[0,+\infty) \mapsto m(r) r^{\alpha-2}\right)
$$

is increasing. 
Proof. - We can consider only the case $\mathcal{M}_{\lambda, \Lambda}^{-}\left(D^{2} u\right) \leqslant 0$, the other one being absolutely analogous.

From inequality (3.12), letting $r_{1} \rightarrow+\infty$ and being $m\left(r_{1}\right) \geqslant 0$, it follows

$$
m(r) \geqslant\left\{\begin{array}{ll}
m\left(r_{2}\right), & \text { if } \beta=2, \\
\frac{m\left(r_{2}\right) r_{2}^{\beta-2}}{r^{\beta-2}}, & \text { if } \beta>2,
\end{array} \quad \forall r \geqslant r_{2}>0 .\right.
$$

Remark 5. - If $u \in C\left(\mathbb{R}^{N}\right)$ is a viscosity solution of

$$
u \geqslant 0, \quad \mathcal{M}_{\lambda, \Lambda}^{-}\left(D^{2} u\right) \leqslant 0 \quad \text { in } \mathbb{R}^{N}
$$

the same holds true for the radial function $m(|x|)=\inf _{B_{|x|}} u$.

Indeed, since the operator $\mathcal{M}_{\lambda, \Lambda}^{-}$is rotations invariant, $u(P x)$ is again a supersolution for every matrix $P$ satisfying $P^{\mathrm{T}}=P^{-1}\left(P^{\mathrm{T}}\right.$ and $P^{-1}$ denote respectively the transposed and the inverse matrices of $P$ ). Then the claim follows observing that the minimum of supersolutions is again a supersolution and that, in view of the Strong Maximum Principle (Theorem 2.2), $m$ can be written as

$$
m(|x|)=\min _{\partial B_{|x|}} u=\min _{P} u\left(P x_{0}\right) \quad \text { for some } x_{0} \text { with }\left|x_{0}\right|=|x| .
$$

An analogous result holds true for subsolutions.

\section{LIOUVILLE THEOREM FOR FULLY NONLINEAR EQUATIONS HAVING ZERO ORDER TERMS}

In this section we are concerned with the Liouville property for viscosity supersolutions of the uniformly elliptic equation

$$
u \geqslant 0, \quad F\left(x, D^{2} u\right)+h(x) u^{p}=0 \quad \text { in } \mathbb{R}^{N},
$$

with $N \geqslant 2$ as usual, where $F$ satisfies (2.1) and (2.2), $h(x)$ is a nonnegative continuous function in $\mathbb{R}^{N}$ and $p$ is a positive exponent.

First of all, we observe that if $u \in C\left(\mathbb{R}^{N}\right)$ is a viscosity supersolution of (4.1), then, by (2.7), $u$ satisfies

$$
u \geqslant 0, \quad \mathcal{M}_{\lambda, \Lambda}^{-}\left(D^{2} u\right)+h(x) u^{p} \leqslant 0 \quad \text { in } \mathbb{R}^{N},
$$


and therefore, $h$ being nonnegative, it results

$$
u \geqslant 0, \quad \mathcal{M}_{\lambda, \Lambda}^{-}\left(D^{2} u\right) \leqslant 0 \quad \text { in } \mathbb{R}^{N}
$$

As it was pointed out in the previous section, in the case $\beta=2$, with $\beta$ defined in (3.8), conditions (4.3) suffice to conclude that $u$ is constant and then, by (4.2), that $u$ is identically equal to zero.

Thus, in the sequel we will always consider the nontrivial case $\beta>2$, in which the zero order term of inequality (4.2) becomes essential: we will identify a range of values for the exponent $p$ which constrains the solution $u$ to vanish identically.

The proof we are going to present essentially makes use of the Comparison Principle and of the Strong Maximum Principle (respectively, Theorems 2.1 and 2.2).

THEOREM 4.1. - Assume that $\beta=\frac{\Lambda}{\lambda}(N-1)+1>2$ and let $u \in$ $C\left(\mathbb{R}^{N}\right)$ be a viscosity solution of

$$
u \geqslant 0, \quad F\left(x, D^{2} u\right)+h(x) u^{p} \leqslant 0 \quad \text { in } \mathbb{R}^{N},
$$

where $h \in C\left(\mathbb{R}^{N}\right)$ is a nonnegative function such that there exist $H$, $r_{0}>0$ and $\gamma \geqslant-2$ satisfying

$$
h(x) \geqslant H|x|^{\gamma} \text { for }|x| \geqslant r_{0} .
$$

If $\gamma=-2$ and $0<p<1$ or if $\gamma>-2$ and $0<p \leqslant(\beta+\gamma) /(\beta-2)$, then

$$
u \equiv 0
$$

Proof. - Let $u$ be as in the statement; by the Strong Maximum Principle if there exists a point where $u$ is zero then $u$ vanishes identically. Thus we will assume, by contradiction, that $u>0$ in $\mathbb{R}^{N}$.

For every $r \geqslant 0$, let us set $m(r)=\min _{|x| \leqslant r} u(x)$ as in Theorem 3.1; observe that, in our assumption, $m$ is strictly positive and, again by Theorem 2.2, it is a strictly decreasing function of $r$.

Moreover, since $u$ in particular satisfies (4.3), by Corollary 3.1 we have

$$
m(r) \leqslant \frac{m(R) R^{\beta-2}}{r^{\beta-2}} \quad \forall R \geqslant r>0 .
$$


Let us now consider the radial function

$$
\zeta(x)=m(r)\left\{1-\frac{\left[(|x|-r)^{+}\right]^{3}}{(R-r)^{3}}\right\},
$$

where $R>r \geqslant r_{0}$ are arbitrarily fixed. Since $\zeta(x) \leqslant 0<u(x)$ for $|x| \geqslant R$ and $\zeta(x) \equiv m(r)<u(x)$ for $|x|<r$, and being $\zeta(x)=u(x)$ for at least one point $x$ with $|x|=r$, the minimum of $u-\zeta$ in $\mathbb{R}^{N}$ is nonpositive and achieved at a certain point $x_{R}^{r}$ such that $r \leqslant\left|x_{R}^{r}\right|<R$.

Applying Definition 2.1 with $\zeta$ as test function and remembering (2.7), from (4.4) it results

$$
\mathcal{M}_{\lambda, \Lambda}^{-}\left(D^{2} \zeta\left(x_{R}^{r}\right)\right)+h\left(x_{R}^{r}\right)\left(u\left(x_{R}^{r}\right)\right)^{p} \leqslant 0 .
$$

On the other hand, by identity (2.5) and by Lemma 3.1, we have, for all $x \in \mathbb{R}^{N} \backslash\{0\}$,

$$
\mathcal{M}_{\lambda, \Lambda}^{-}\left(D^{2} \zeta(x)\right)=-\frac{3 \Lambda m(r)}{(R-r)^{3}}\left[2+(N-1) \frac{(|x|-r)^{+}}{|x|}\right](|x|-r)^{+},
$$

and therefore, using also assumption (4.5) and the fact $\left|x_{R}^{r}\right| \geqslant r \geqslant r_{0}$, we get

$$
\begin{aligned}
\left(u\left(x_{R}^{r}\right)\right)^{p} \leqslant & \frac{3 \Lambda H^{-1} m(r)}{(R-r)^{3}}\left[2+(N-1) \frac{\left(\left|x_{R}^{r}\right|-r\right)^{+}}{\left|x_{R}^{r}\right|}\right] \\
& \times\left(\left|x_{R}^{r}\right|-r\right)^{+}\left|x_{R}^{r}\right|^{-\gamma} .
\end{aligned}
$$

If $\left|x_{R}^{r}\right|=r$, we immediately reach the contradiction $u\left(x_{R}^{r}\right)=0$; therefore we can assume $r<\left|x_{R}^{r}\right|<R$ and, by (4.7), it results

$$
\left(u\left(x_{R}^{r}\right)\right)^{p} \leqslant \frac{3 \Lambda(N+1)}{H} \frac{\left|x_{R}^{r}\right|^{-\gamma} m(r)}{(R-r)^{2}} .
$$

Being $u\left(x_{R}^{r}\right) \geqslant m(R)$ and $r<\left|x_{R}^{r}\right|<R$, we then have

$$
m(R) \leqslant C \frac{R^{\gamma^{-} / p} m(r)^{1 / p}}{r^{\gamma^{+} / p}(R-r)^{2 / p}},
$$

for all $R>r \geqslant r_{0}$, with $C=[3 \Lambda(N+1) / H]^{1 / p}$.

Combining (4.8) with (4.6) we get

$$
m(R) \leqslant C \frac{R^{\left(\gamma^{-}+\beta-2\right) / p} m(R)^{1 / p}}{r^{\left(\gamma^{+}+\beta-2\right) / p}(R-r)^{2 / p}} \quad \forall R>r \geqslant r_{0},
$$


from which, choosing $r=R / 2$, it follows

$$
m(R) \leqslant C \frac{m(R)^{1 / p}}{R^{(\gamma+2) / p}} \quad \forall R>2 r_{0},
$$

where the same $C$ denotes from now on different positive constants. We have now to distinguish several cases.

Assume first that $\gamma=-2$ and $0<p<1$; from (4.9) we deduce that

$$
m(R) \geqslant C \quad \forall R>2 r_{0} .
$$

It then follows that the infimum

$$
\mu=\inf _{R>0} m(R)=\lim _{R \rightarrow+\infty} m(R)
$$

is strictly positive. Let us set $v(x)=u(x)-\mu$; thus, $v \in C\left(\mathbb{R}^{N}\right)$ is still a viscosity solution of (4.4), as it is immediate to verify. Applying the above arguments to $v$, we obtain that the function $m_{v}(r)=\min _{|x| \leqslant r} v(x)$ satisfies

$$
m_{v}(R) \geqslant C \quad \forall R>2 r_{0},
$$

accordingly to (4.10). But this evidentely contradicts the fact that

$$
\lim _{R \rightarrow+\infty} m_{v}(R)=\lim _{R \rightarrow+\infty}(m(R)-\mu)=0 .
$$

Assume now that $\gamma>-2$ and $0<p \leqslant(\beta+\gamma) /(\beta-2)$.

If $0<p \leqslant 1$, from (4.9) we immediately get the contradiction

$$
R^{(\gamma+2) / p} \leqslant C m(R)^{(1-p) / p} \leqslant C m(0)^{(1-p) / p} \quad \forall R>2 r_{0} .
$$

If this is not the case, then $p>1$ and from (4.9) we obtain

$$
m(R) \leqslant \frac{C}{R^{(\gamma+2) /(p-1)}} \quad \forall R>2 r_{0},
$$

which in turn implies

$$
R^{\beta-2} m(R) \leqslant \frac{C}{R^{\frac{\gamma+2}{p-1}-\beta+2}} \quad \forall R>2 r_{0} .
$$

If $p<(\beta+\gamma) /(\beta-2)$, then $(\gamma+2) /(p-1)-\beta+2>0$ and the fact that the positive and increasing function $R^{\beta-2} m(R)$ tends to 0 as $R \rightarrow+\infty$ gives the desired contradiction. 
Thus, it remains to consider only the limit case $p=(\beta+\gamma) /(\beta-2)$. In such a case, from (4.11) we deduce that the function $R^{\beta-2} m(R)$ is bounded from above; we will show that this information, combined with (4.4), again leads to a contradiction. Fixed $R_{1} \geqslant r_{0}, \gamma_{1}>0$ and $\gamma_{2} \in \mathbb{R}$, for $x$ such that $|x| \geqslant R_{1}$, let us define the radial function

$$
\Gamma(x)=\gamma_{1} \frac{\log (1+|x|)}{|x|^{\beta-2}}+\gamma_{2} .
$$

The choice $R_{1} \geqslant \exp ((2 \beta-3) /(\beta-2)(\beta-1))-1$ makes $\Gamma$ a convex decreasing function of $|x|$; in such a case, using Lemma 3.1 and definitions (2.5) and (3.8), we can easily obtain:

$$
\begin{aligned}
\mathcal{M}_{\lambda, \Lambda}^{-}\left(D^{2} \Gamma(x)\right) & =-\lambda \gamma_{1}\left[\frac{\beta-3}{(1+|x|)|x|^{\beta-1}}+\frac{1}{(1+|x|)^{2}|x|^{\beta-2}}\right] \\
& \geqslant-\frac{\lambda \gamma_{1}(\beta-2)}{|x|^{\beta}},
\end{aligned}
$$

for all $x$ such that $|x| \geqslant R_{1}$.

We now arbitrarily choose a radius $R_{2}>R_{1}$ and fix the constants $\gamma_{1}>0$ and $\gamma_{2} \in \mathbb{R}$ in such a way that

$$
\Gamma\left(R_{1}\right) \leqslant m\left(R_{1}\right) \quad \text { and } \quad \Gamma\left(R_{2}\right)=m\left(R_{2}\right) ;
$$

this means that we have to pick $\gamma_{1}$ in the interval

$$
0<\gamma_{1} \leqslant\left(m\left(R_{1}\right)-m\left(R_{2}\right)\right) /\left(\frac{\log \left(1+R_{1}\right)}{R_{1}^{\beta-2}}-\frac{\log \left(1+R_{2}\right)}{R_{2}^{\beta-2}}\right),
$$

which is possible since

$$
R_{1} \geqslant \exp \left(\frac{2 \beta-3}{(\beta-2)(\beta-1)}\right)-1>\exp \left(\frac{1}{\beta-2}\right)-1,
$$

and then define

$$
\gamma_{2}=m\left(R_{2}\right)-\gamma_{1} \frac{\log \left(1+R_{2}\right)}{R_{2}^{\beta-2}}
$$

We then have

$$
\Gamma \leqslant u \quad \text { on }\left\{|x|=R_{1}\right\} \cup\left\{|x|=R_{2}\right\}
$$


and, moreover, by the previous calculation,

$$
\mathcal{M}_{\lambda, \Lambda}^{-}\left(D^{2} \Gamma(x)\right) \geqslant-\frac{\gamma_{1} \lambda(\beta-2)}{|x|^{\beta}} \quad \text { in }\left\{R_{1}<|x|<R_{2}\right\} .
$$

On the other hand, by (4.6), for all $x$ such that $|x| \geqslant R_{1}, u$ satisfies

$$
u(x) \geqslant m(|x|) \geqslant \frac{m\left(R_{1}\right) R_{1}^{\beta-2}}{|x|^{\beta-2}},
$$

and then, from (4.4) with $p=(\beta+\gamma) /(\beta-2)$, and from (2.7) and (4.5), if $R_{1} \geqslant r_{0}$ it follows that

$$
\mathcal{M}_{\lambda, \Lambda}^{-}\left(D^{2} u\right) \leqslant-\frac{H\left(m\left(R_{1}\right) R_{1}^{\beta-2}\right)^{(\beta+\gamma) /(\beta-2)}}{|x|^{\beta}} \text { in }\left\{R_{1}<|x|<R_{2}\right\}
$$

in the viscosity sense. From (4.13) and (4.15), choosing, if necessary, a smaller $\gamma_{1}>0$, we deduce

$$
\mathcal{M}_{\lambda, \Lambda}^{-}\left(D^{2} u(x)\right) \leqslant-\frac{C}{|x|^{\beta}} \leqslant \mathcal{M}_{\lambda, \Lambda}^{-}\left(D^{2} \Gamma(x)\right) \quad \text { in }\left\{R_{1}<|x|<R_{2}\right\},
$$

for some constant $C>0$. This, combined with (4.12), allows us to apply Theorem 2.1 and conclude

$$
\Gamma \leqslant u \quad \text { in }\left\{R_{1} \leqslant|x| \leqslant R_{2}\right\}
$$

for any $R_{2}>R_{1}$. Letting $R_{2} \rightarrow+\infty$, being $\gamma_{2} \rightarrow 0$, we obtain the existence of a positive constant $\tilde{\gamma}_{1}$ such that

$$
u(x) \geqslant \tilde{\gamma_{1}} \frac{\log (1+|x|)}{|x|^{\beta-2}}
$$

for all $|x| \geqslant R_{1}$. This implies that

$$
R^{\beta-2} m(R) \geqslant \tilde{\gamma_{1}} \log (1+R) \quad \forall R \geqslant R_{1},
$$

which contradicts the obtained upper bound on the function $R^{\beta-2} m(R)$.

Remark 6. - The previous theorem extends to the nonlinear operators the analogous result already known for the Laplacian (see $[10,12]$ ). 
Moreover, it also includes the case $0<p \leqslant 1$ and the case $\gamma=-2$ for the Laplace operator, which was not covered by $[10,12]$.

Remark 7. - Theorem 4.1 provides the optimal result.

Indeed, the problem

$$
u \geqslant 0, \quad \mathcal{M}_{\lambda, \Lambda}^{-}\left(D^{2} u\right)+\left(1+|x|^{2}\right)^{\gamma / 2} u^{p} \leqslant 0 \quad \text { in } \mathbb{R}^{N}
$$

admits a nontrivial classical (and therefore viscosity) solution in the cases $p>0$ if $\gamma<-2, p \geqslant 1$ if $\gamma=-2$ and $p>(\beta+\gamma) /(\beta-2)$ if $\gamma>-2$. To see this, let us consider functions $u_{\delta}$ of the form

$$
u_{\delta}(x)=\frac{C_{\delta}}{\left(1+|x|^{2}\right)^{\delta}} \quad x \in \mathbb{R}^{N},
$$

with $\delta, C_{\delta}>0$. A direct computation shows that, for all $x$ such that $|x|^{2} \leqslant 1 /(2 \delta+1), u_{\delta}$ is a concave and decreasing function of $|x|$ and, taking into account that $\Lambda(N-1)=\lambda(\beta-1)$, it results

$$
\begin{aligned}
\mathcal{M}_{\lambda, \Lambda}^{-}\left(D^{2} u_{\delta}(x)\right) & =-2 \Lambda C_{\delta} \delta\left[\frac{(N-1)}{\left(1+|x|^{2}\right)^{\delta+1}}-\frac{(2 \delta+1)|x|^{2}-1}{\left(1+|x|^{2}\right)^{\delta+2}}\right] \\
& \leqslant-\frac{2 \Lambda(N-1) C_{\delta} \delta}{\left(1+|x|^{2}\right)^{\delta+1}}=-\frac{2 \lambda(\beta-1) C_{\delta} \delta}{\left(1+|x|^{2}\right)^{\delta+1}} .
\end{aligned}
$$

If $|x|^{2} \geqslant 1 /(2 \delta+1)$, then $u_{\delta}$ is a decreasing and convex function of $|x|$ and satisfies

$$
\begin{aligned}
& \mathcal{M}_{\lambda, \Lambda}^{-}\left(D^{2} u_{\delta}(x)\right) \\
& \quad=-2 \lambda C_{\delta} \delta\left[\frac{\beta-1}{\left(1+|x|^{2}\right)^{\delta+1}}-\frac{(2 \delta+1)|x|^{2}-1}{\left(1+|x|^{2}\right)^{\delta+2}}\right] \\
& =-2 \lambda C_{\delta} \delta \frac{(\beta-2(\delta+1))|x|^{2}+\beta}{\left(1+|x|^{2}\right)^{\delta+2}} \leqslant-\frac{2 \lambda C_{\delta} \delta(\beta-2(\delta+1))}{\left(1+|x|^{2}\right)^{\delta+1}} .
\end{aligned}
$$

Therefore, in both cases, we have

$$
\begin{aligned}
& \mathcal{M}_{\lambda, \Lambda}^{-}\left(D^{2} u_{\delta}(x)\right)+\left(1+|x|^{2}\right)^{\frac{\gamma}{2}}\left[u_{\delta}(x)\right]^{p} \\
& \quad \leqslant-\frac{2 \lambda C_{\delta} \delta(\beta-2(\delta+1))}{\left(1+|x|^{2}\right)^{\delta+1}}+\frac{C_{\delta}^{p}}{\left(1+|x|^{2}\right)^{p \delta-\frac{\gamma}{2}}} .
\end{aligned}
$$


It is easily seen that the right hand side is nonpositive if the constants $\delta$ and $C_{\delta}$ satisfy

$$
\left\{\begin{array}{l}
2(\delta+1)<\beta \\
\delta+1 \leqslant p \delta-\frac{\gamma}{2} \\
C_{\delta}^{p-1} \leqslant 2 \lambda \delta(\beta-2(\delta+1))
\end{array}\right.
$$

and we can find constants $\delta, C_{\delta}>0$ simultaneously satisfying the above conditions exactly in the cases $\gamma<-2$ and $p>0, \gamma=-2$ and $p \geqslant 1$, $\gamma>-2$ and $p>(\beta+\gamma) /(\beta-2)$.

Remark 8. - If in Theorem 4.1 we add assumptions on the operator $F$, then we can obtain an accordingly extended range of admissible values for the exponent $p$ which guarantees the Liouville property for problem (4.4).

The most favourable case occurs when $F=\mathcal{M}_{\lambda, \Lambda}^{+}$; indeed, as a first fact, one observes that if $u \in C\left(\mathbb{R}^{N}\right)$ is a viscosity solution of

$$
u \geqslant 0, \quad \mathcal{M}_{\lambda, \Lambda}^{+}\left(D^{2} u\right)+h(x) u^{p} \leqslant 0 \quad \text { in } \mathbb{R}^{N},
$$

then $u$ satisfies

$$
u \geqslant 0, \quad \lambda \Delta u+h(x) u^{p} \leqslant 0 \quad \text { in } \mathbb{R}^{N}
$$

in the viscosity sense and therefore $u \equiv 0$ provided that $0<p \leqslant$ $(N+\gamma) /(N-2)$ if $\gamma>-2$ and that $0<p<1$ if $\gamma=-2$. More than that, by Corollary 3.1 we know that the function $r \in[0,+\infty) \mapsto$ $m(r) r^{\alpha-2}$ is increasing, with $\alpha=\frac{\lambda}{\Lambda}(N-1)+1$, and, applying exactly the same arguments of the proof of Theorem 4.1, we conclude that $u \equiv 0$ under the assumption

$$
\begin{array}{ll}
0<p<1 & \text { if } \gamma=-2, \\
0<p \leqslant(\alpha+\gamma) /(\alpha-2) & \text { if } \gamma>-2 .
\end{array}
$$

Remark 9. - It is worth observing that a Liouville type result for problem (4.4) could be obtained following an alternative procedure. Indeed, it is known (see [8]) that if $u \in C\left(B_{2 R}\right)$ is a viscosity solution of

$$
u \geqslant 0, \quad \mathcal{M}_{\lambda, \Lambda}^{-}\left(D^{2} u\right) \leqslant 0 \quad \text { in } B_{2 R},
$$

where $B_{2 R}$ is a ball of radius $2 R$ for a given $R>0$, then there exist positive constants $q_{0}$ and $C$, depending only on $N$ and on the ellipticity 
constants $\lambda$ and $\Lambda$, such that $u$ satisfies the following so called weak Harnack inequality:

$$
\operatorname{meas}\left(B_{R / 2} \cap\{u>t\}\right) \leqslant \frac{C R^{N}}{t^{q_{0}}}\left(\min _{B_{R}} u\right)^{q_{0}} \quad \forall t>0,
$$

with $\{u>t\}=\left\{x \in B_{2 R}: u(x)>t\right\}$ and meas $(E)$ equals to the Lebesgue measure of the measurable set $E \subset \mathbb{R}^{N}$.

If $\beta>2$, testing the previous inequality on the particular solution particular solution $u$ defined by (3.19), produces the estimate

$$
q_{0} \leqslant \frac{N}{\beta-2}
$$

already found in [23]. In the particular case in which $\beta=N$, that is when $\Lambda=\lambda$ and the extremal operator $\mathcal{M}_{\lambda, \Lambda}^{-}$coincides with the Laplace operator (up to the positive factor $\lambda$ ), inequality (4.18) reduces to equality. Indeed, superharmonic functions satisfy the weak Harnack inequality (4.17) with the exponent $q_{0}=N /(N-2)$. A proof of this fact can be found in [19].

We conjecture that equality holds in (4.18) also in the general case $\beta>N$.

Let us observe at this purpose that for the supersolution $m(|x|),(4.17)$ holds true with the exponent $q_{0}=N /(\beta-2)$. Indeed, Corollary 3.1 yields:

$$
\begin{aligned}
& \operatorname{meas}\left(B_{R / 2} \cap\{m(|x|)>t\}\right) \\
& \leqslant \operatorname{meas}\left(B_{R / 2} \cap\left\{\frac{m(R) R^{\beta-2}}{|x|^{\beta-2}}>t\right\}\right) \\
& \leqslant \frac{C R^{N}}{t^{N /(\beta-2)}}(m(R))^{N /(\beta-2)} \quad \forall t>0 .
\end{aligned}
$$

Let now $u \in C\left(\mathbb{R}^{N}\right)$ be a viscosity solution of (4.4) with $p>1$ and, arguing by contradiction, assume that $u>0$ in $\mathbb{R}^{N}$. Since $u$ satisfies (4.17) and (4.11), for $R$ large enough we obtain

$$
\operatorname{meas}\left(B_{R / 2} \cap\{u>t\}\right) \leqslant C \frac{R^{N-\frac{q_{0}(2+\gamma)}{p-1}}}{t^{q_{0}}} .
$$


Let $R \rightarrow+\infty$ and observe that

$$
\{u>t\}=\bigcup_{R>0}\left(B_{R / 2} \cap\{u>t\}\right)
$$

and that $\left\{B_{R / 2} \cap\{u>t\}\right\}$ is an increasing family of open sets. If $p<$ $1+\left(q_{0}(2+\gamma) / N\right)$ then we find

$$
\operatorname{meas}(\{u>t\})=0 \quad \forall t>0
$$

which implies $u \equiv 0$.

If $p=1+\left(q_{0}(2+\gamma) / N\right)$, from the above we obtain

$$
\operatorname{meas}(\{u>t\}) \leqslant \frac{C}{t^{q_{0}}} \quad \forall t>0,
$$

which means that $u$ belongs to the Marcinkiewicz space $M^{q_{0}}\left(\mathbb{R}^{N}\right)$. This implies, by (4.14), that the function $m\left(R_{1}\right) R_{1}^{\beta-2} /|x|^{\beta-2}$ belongs to $M^{q_{0}}\left(\mathbb{R}^{N} \backslash B_{R_{1}}\right)$, for every fixed $R_{1}>0$.

In particular, for every $R>R_{1}$, it follows that

$$
\begin{gathered}
\operatorname{meas}\left(\left\{\frac{m\left(R_{1}\right) R_{1}^{\beta-2}}{|x|^{\beta-2}}>\frac{m\left(R_{1}\right) R_{1}^{\beta-2}}{R^{\beta-2}}\right\} \backslash B_{R_{1}}\right) \\
=C\left(R^{N}-R_{1}^{N}\right) \leqslant C\left(\frac{R^{\beta-2}}{m\left(R_{1}\right) R_{1}^{\beta-2}}\right)^{q_{0}}
\end{gathered}
$$

which implies

$$
q_{0} \geqslant \frac{N}{\beta-2} .
$$

Remembering (4.18), if $q_{0}<N /(\beta-2)$, then we immediately have a contradiction and we conclude that $u \equiv 0$. Otherwise, $q_{0}=N /(\beta-2)$ and thus

$$
p=1+\frac{q_{0}(2+\gamma)}{N}=\frac{\beta+\gamma}{\beta-2} .
$$

In this case, reasoning as in the proof of Theorem 4.1, we obtain inequality (4.16). Since the function $\tilde{\gamma}_{1}\left(\log (1+|x|) /|x|^{\beta-2}\right)$ lies below $u$ in $\mathbb{R}^{N} \backslash B_{R_{1}}$ but it does not belong to the Marcinkievicz space $M^{\frac{N}{\beta-2}}\left(\mathbb{R}^{N} \backslash\right.$ $B_{R_{1}}$ ), we again obtain a contradiction. 
Thus, by means of the weak Harnack inequality applied to $u$, we obtain the Liouville property for problem (4.4) under the assumption

$$
0<p \leqslant 1+\frac{(2+\gamma) q_{0}}{N} .
$$

In the semilinear case, being $q_{0}=N /(N-2)$, the result is optimal; in the fully nonlinear case, we have the same conclusion if and only if

$$
q_{0}=\frac{N}{\beta-2} .
$$

On the other hand, reasoning on $m(|x|)$ and applying (4.19) instead of (4.17) we may obtain the same conclusions as those of Theorem 4.1. Indeeed, from (4.19) and (4.11) we get that the function $m$ vanishes identically in the case $p<(\beta+\gamma) /(\beta-2)$ whereas it belongs to the Marcinkievicz space $M^{\frac{N}{\beta-2}}\left(\mathbb{R}^{N}\right)$ if $p=(\beta+\gamma) /(\beta-2)$. In the first case we immediately obtain the contradiction, in the last case the contradiction follows from the fact that $m$ lies above the function $\tilde{\gamma}_{1}(\log (1+|x|)) /\left(|x|^{\beta-2}\right)$ which does not belong to $M^{\frac{N}{\beta-2}}\left(\mathbb{R}^{N} \backslash B_{R_{1}}\right)$.

\section{ACKNOWLEDGEMENT}

The authors wish to thank Professor Italo Capuzzo Dolcetta for good advice and useful discussions.

\section{REFERENCES}

[1] Bahri A., Lions P.L., Solutions of superlinear elliptic equations and their Morse indexes, Comm. Pure Appl. Math. 45 (1992) 1205-1215.

[2] Berestycki H., Capuzzo Dolcetta I., Nirenberg L., Problèmes elliptiques indéfinis et théorèmes de Liouville non-linéaires, C. R. Acad. Sci. Paris, Série I 317 (1993) 945-950.

[3] Berestycki H., Capuzzo Dolcetta I., Nirenberg L., Superlinear indefinite elliptic problems and nonlinear Liouville theorems, Topol. Methods Nonlinear Anal. 4 (1994) 59-78.

[4] Birindelli I., Capuzzo Dolcetta I., Cutrì A., Liouville theorems for semilinear equations on the Heisenberg group, Ann. Inst. Henri Poincaré, Analyse Non Linéaire 14 (3) (1997) 295-308.

[5] Birindelli I., Capuzzo Dolcetta I., Cutrì A., Indefinite semi-linear equations on the Heisenberg group: a priori bounds and existence, Comm. Partial Differential Equations 23 (7-8) (1998) 1123-1157. 
[6] Birindelli I., Mitidieri E., Liouville theorems for elliptic inequalities and applications, Proc. Royal Soc. Edinburgh 128A (1998) 1217-1247.

[7] Caffarelli L.A., Interior a priori estimates for solutions of fully nonlinear equations, Ann. Math. 130 (1989) 189-213.

[8] Caffarelli L.A., Cabré X., Fully Nonlinear Elliptic Equations, American Mathematical Society Colloquium Publications, Vol. 43, AMS, Providence, RI, 1995.

[9] Capuzzo Dolcetta I., Teoremi di Liouville e stime a priori per equazioni ellittiche semilineari, Rend. Sem. Mat. Fis. Milano, to appear.

[10] Capuzzo Dolcetta I., Cutrì A., On the Liouville property for sub-laplacians, Ann. Scuola Norm. Sup. Pisa, Cl. Sci. (4) 25 (1-2) (1997) 239-256.

[11] Crandall M.G., Ishii H., Lions P.L., User's guide to viscosity solutions of second order partial differential equations, Bull. Amer. Math. Soc. 27 (1992) 1-67.

[12] Cutrì A., Problemi semilineari ed integro-differenziali per sublaplaciani, Ph.D. Thesis, Universitá di Roma Tor Vergata, 1997.

[13] Gidas B., Spruck J., A priori bounds for positive solutions of nonlinear elliptic equations, Comm. PDE 8 (1981) 883-901.

[14] Gidas B., Spruck J., Global and local behavior of positive solutions of nonlinear elliptic equations, Comm. Pure Appl. Math. 35 (1981) 525-598.

[15] Gilbarg D., Trudinger N.S., Elliptic Partial Differential Equations of Second Order, 2nd ed., Springer, Berlin, 1983.

[16] Ishii H., Lions P.L., Viscosity solutions of fully nonlinear second order elliptic partial differential equations, J. Differential Equations 83 (1990) 26-78.

[17] Hörmander L., Notions of Convexity, Progress in Mathematics, Vol. 127, Birkhäuser, Boston, MA, 1994.

[18] Lanconelli E., Uguzzoni F., Asymptotic behaviour and non existence theorems for semilinear Dirichlet problems involving critical exponent on unbounded domains of the Heisenberg group, Boll. Unione Mat. Ital., Sez B 8 (1) (1998).

[19] Leoni F., work in preparation.

[20] Pucci C., Operatori ellittici estremanti, Ann. Mat. Pura Appl. 72 (1966) 141-170.

[21] Protter M.H., Weinberger H.F., Maximum Principles in Differential Equations, Prentice Hall, 1967.

[22] Ramos M., Terracini S., Troestler C., Problèmes elliptiques surlinéaires avec nonlinéarité sans signe défini, C. R. Acad. Sci. Paris, Série I 325 (1997) 283-286.

[23] Trudinger N.S., Lectures on nonlinear elliptic equations of second order, in: Lectures in Mathematical Sciences, The University of Tokyo, 1995.

[24] Uguzzoni F., A Liouville-type theorem on halfspaces for the Kohn laplacian, Proc. Amer. Math. Soc. 127 (1) (1999) 117-123.

[25] Uguzzoni F., A non existence theorem for a semilinear Dirichlet problem involving critical exponent on halfspaces of the Heisenberg group, NODEA 6 (2) (1999). 